\title{
Issues in implementing prenatal screening for cystic fibrosis: Results of a working conference
}

James E. Haddow, $M D^{1}$, Linda A. Bradley, PhD ${ }^{1}$, Glenn E. Palomaki, BS ${ }^{1}$, Richard A. Doherty, $M D^{1}$, Barbara A. Bernhardt, MS', David J. H. Brock, PhD ${ }^{3}$, Brian Cheuvront, PhD ${ }^{4}$, George C. Cunningham, MD", Alan E. Donnenfeld, $M D^{6}$, Jeryl L. Erickson, $M S^{1}$, Henry A. Erlich, PhD ${ }^{8}$, Richard M. Ferrie, $P h D^{9}$,

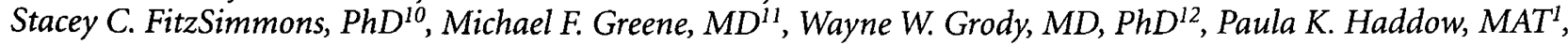
Hilary Harris, $M D^{12}$, Lewis B. Holmes, $M D^{13}$, R. Rodney Howell, $M D^{14}$, Michael Katz, $M D^{15}$, Katherine W. Klinger, PhD ${ }^{16}$, Edward M. Kloza, $M S^{1}$, Michael L. LeFevre, MD, MSPH ${ }^{17}$, Stephen Little, PhD ${ }^{9}$, Greg Loeben, $P h D^{18}$, Margaret McGovern, $M D, P h D^{7}$, Reed E. Pyeritz, $M D$, PhD ${ }^{19}$, Peter T. Rowley, $M D^{20}$, Randall K. Saiki, BS , M. Priscilla Short, $M D^{21}$, John Tabone, $P h D^{23}$, Nicholas J. Wald, FRCP ${ }^{22}$, Nachama L. Wilker, $B A^{23}$, David R. Witt, $M D^{24}$

\begin{abstract}
Purpose: To summarize a conference convened to examine how cystic fibrosis screening might appropriately be introduced into routine prenatal practice. Methods: Participants included experts from various relevant disciplines. Systematic reviews and data from individual trials were presented; issues were identified and discussed. Results: Judged by published criteria, prenatal cystic fibrosis screening is suitable for introduction. Screening can be performed cost-effectively by identifying racial/ethnic groups at sufficient risk and then using either of two models for delivering laboratory services. Validated educational materials exist. Ethical issues are not unique. Conclusions: Once adequate facilities for patient and provider education, testing, counseling, quality control, and monitoring are in place, individual programs can begin prenatal screening for cystic fibrosis.
\end{abstract}

Genetics in Medicine, 1999:1(4):129-135.

Key words: cystic fibrosis, screening, prenatal, genetic testing

\section{BACKGROUND}

The sixth Scarborough Conference (July 26-28, 1998) was convened to examine how prenatal screening for cystic fibrosis might most appropriately be introduced into routine prac-

From the 'Foundation for Blood Research, Scarborough. Maine: 'Johns Hopkins School of Medicine, Baltimore, Maryland; ${ }^{3}$ University of Edinburgh, Edinburgh. Scotland; ${ }^{4}$ University of North Carolina, Chapel Hill, North Carolina; ${ }^{5}$ California Departinent of Health, Berkeley, California; 'Pennsylvania Hospital, Philadelphia, Pennsylivania; ${ }^{7}$ Roche Molecular Systems, Alameda, California; ${ }^{8} Z$ Zeneca Diagnostics, Cheshire, England; ${ }^{9} \mathrm{Cystic} F i b r o-$ sis Foundation, Bethesda, Maryland: ${ }^{10}$ Vincent Memorial Obstetric Association, Boston, Massachusetts; "UCLA School of Medicine, Los Angeles, California; ${ }^{12}$ Central Manchester Health Care Trust, Manchester, England; ${ }^{13}$ Massachusetts General Hospital, Boston, Massachusetts; 14 University of Miami School of Medicine, Miami, Florida; ${ }^{15}$ March of Dimes Birth Defects Foundation, White Plains, New York; "Genzyme, Framingham, Massachusetts; "University of Missouri-Columbia, Columbia, Missouri; "'University' of Pennsylvania, Philadelphia, Pennsylvania; ${ }^{19}$ Mount Sinai School of Medicine. New' York, New' York; ${ }^{20}$ Allegheny University of the Health Sciences, Pittsburgh, Pennsylvania: ${ }^{21}$ University of Rochester School of Medicine, Rochester, New York; ${ }^{22}$ University of Chicago, Chicago, Illinois; ${ }^{23}$ Rapigene, Inc., Bothell, Washington; ${ }^{24}$ Wolfson Institute for Preventive Medicine, London, England; ${ }^{25}$ Alliance of Genetic Support Groups, Washington, $D C$; and ${ }^{26}$ Kaiser Permanente, San Jose, California

James E. Haddow, MD, Foundation for Blood Research, P.O. Box 190, Scarborough, Maine 04070-0190; E-mail: jbeaudoi@fbr.org

The meeting was organized and the manuscript prepared by James E. Haddow, Lindn A. Bradley, Glenn E. Palomaki, and Richard A. Doherty.

Conclusions drawn in the paper do not necessarily represent a consensus of all who participated in the discussion or the positions of the organizations and institutions represented by the authors.

Submitted for consideration January 21, 1999; accepted for publication April 6, 1999. tice. This meeting followed the April, 1997 report of the NIH Consensus Development Conference expert panel that recommended implementation of prenatal and preconceptional screening for cystic fibrosis in the United States. ${ }^{1}$ In response to those recommendations, the American College of Medical Genetics and the American College of Obstetrics and Gynecology issued statements that routinely offering prenatal genetic testing for cystic fibrosis was not standard of practice and would not be until certain specified components, such as patient and provider education and definitions of minimum laboratory requirements, were in place. ${ }^{2,3}$

The 36 participants at the sixth Scarborough Conference included clinicians, consumers, educators, epidemiologists, geneticists, genetic counselors, laboratory scientists, manufacturers of diagnostic products, medical ethicists, public health experts, and representatives of professional organizations. The meeting placed emphasis on obtaining information from leaders of prenatal cystic fibrosis screening trials. An early goal of the meeting was to delineate clearly the two models commonly used in managing the operational aspects of prenatal cystic fibrosis screening and then to present the findings of trials that used these models. Data from screening trials were presented by investigators from the United States and the United Kingdom. This was followed by presentations and discussions on economics and other specific aspects of screening (e.g., laboratory issues, proficiency testing and quality assurance, patient and provider 
education, and consent). The following is a summary report of the deliberations.

\section{SCREENING MODELS}

The aim of prenatal screening for cystic fibrosis is the detection of affected fetuses. Carriers of a recessive trait (such as cystic fibrosis) do not suffer adverse health consequences. Therefore, carrier identification simply represents one critical component of the screening process, whether or not screened individuals are informed of their carrier status. Nearly all of the prenatal cystic fibrosis screening trials used either a one-step (couple) ${ }^{4}$ or a two-step (sequential) model. In the one-step model, both the woman and her partner opt for screening at the outset and both must submit samples before any laboratory testing is initiated. Testing is performed on one of the samples; if this reveals an identifiable mutation, the partner's sample is tested. The couple's test result is considered positive only when both carry identifiable mutations. These couples are then offered counseling and prenatal diagnostic testing. When only the first partner tested is identified as having a mutation, the result is reported as screen negative and information is not routinely provided about carrier status. The two-step model calls for mutation analysis to be performed initially on the woman, with counseling offered whenever a mutation is identified. The male partners of women with identified mutations are then offered counseling and testing. When both partners carry identifiable mutations, they are offered further counseling and diagnostic testing of the fetus.

Figure 1 shows the results of using these models in a hypothetical population of 250,000 pregnant non-Hispanic Caucasian women, assuming complete participation at each step.
In this population, if the prevalence of cystic fibrosis were $1: 2500$, then 100 fetuses would be affected and 249,900 would be unaffected. The laboratory panel is assumed to contain enough mutations to identify $85 \%$ of carriers. Using the twostep model, 8500 of the women (3.4\%) are identified with mutations and 85 of them carry an affected fetus. All of these women receive counseling and are told of their mutation status. The partners of these 8500 women are then offered testing, and 289 of the fathers are found to carry an identifiable mutation. Diagnostic testing in these 289 couples identifies 72 fetuses with cystic fibrosis. Using the one-step model, all of the couples submit their samples together at the outset, but only the 289 couples $(0.1 \%)$ who both carry an identifiable mutation are classified as having a positive test result and offered counseling and diagnostic testing. The same 72 affected fetuses are identified. The one-step model does not include the intermediate step that provides routine reporting of carrier status and counseling services to the 8211 couples where only one partner has an identifiable mutation $(85-72+8415-217$ from Figure 1).

\section{SUMMARY OF PRENATAL CYSTIC FIBROSIS SCREENING TRIALS}

The 19 published reports of prenatal cystic fibrosis screening trials were reviewed. ${ }^{5-23}$ The trials were conducted at 16 sites in the United States and Europe. The one-step model was used by four sites in the United Kingdom and one site in the United States $^{5-14}$ to screen a total of 37,178 couples. The two-step model was used by 11 sites (four each in the United Kingdom and the United States and three elsewhere in Europe $)^{5,7,10-12,15-23}$ to screen a total of 35,469 couples. Collectively, these trials showed that:

\section{Two-step Model}

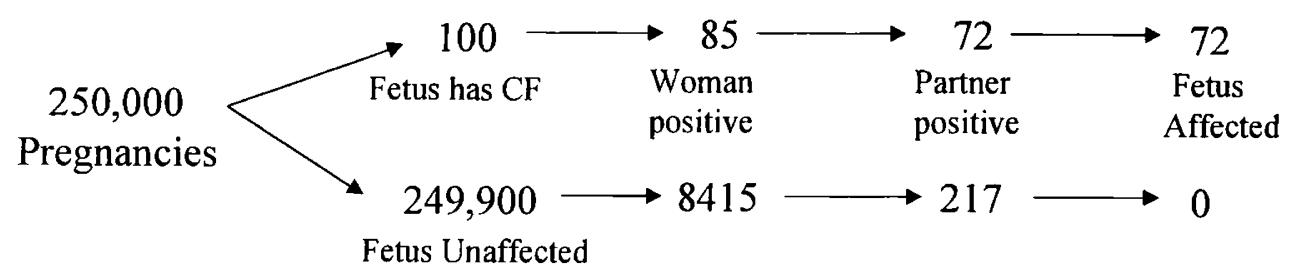

One-step Model

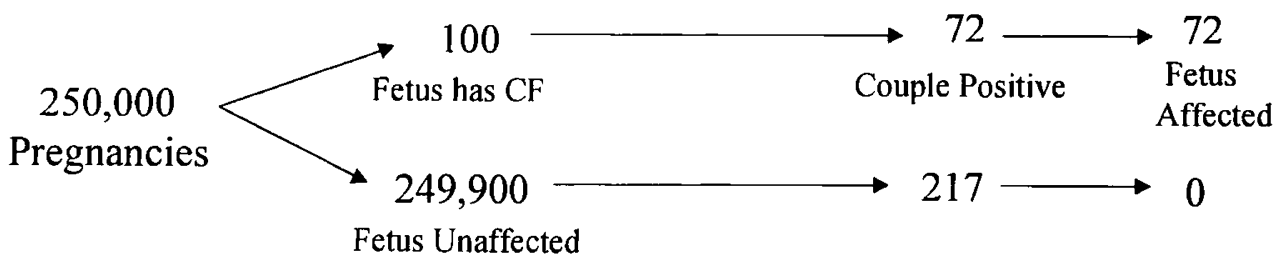

Fig. 1 Prenatal cystic fibrosis screening models. The results of using two cystic fibrosis prenatal screening models in a hypothetical population of 250,000 non-Hispanic Caucasian couples. Assumptions: prevalance is 1:2500 ( 100 affected and 249,900 unaffected pregnancies), $85 \%$ of carriers identified, and $100 \%$ participation. In the two-step model (upper half of the figure), 8500 women ( 85 affected and 8415 unaffected pregnancies) are identified with mutations. These women receive genetic counseling and their partners are offered testing; 289 fathers are found to carry an identifiable mutation. Diagnostic testing in these 289 couples identifies 72 fetuses with cystic fibrosis (one in four). Thirteen fetuses with cystic fibrosis are present among the 8211 couples where only the woman has an identifiable mutation. In the one-step model (bottom half of the figure), all 250,000 women and partners submit samples. Only the 289 couples who both carry an identifiable mutation are classified as having a positive test result and offered genetic counseling and diagnostic testing. The same 72 affected fetuses are identified. 
1) either blood or buccal samples could be used successfully; 2) patient education could be accomplished in both centralized ${ }^{16,20,22}$ and decentralized settings ${ }^{10-14,18}$ in approximately 10 minutes; 3 ) initial uptake rates were high using either model in both the United States trials (average 69\%; range 57 to $78 \%$ ) and in the European trials (average $78 \%$; range 62 to $99 \%$ ); 4) partner compliance rates were high when women were identified as carriers in two-step screening ( $88 \%$ in the United States and $97 \%$ elsewhere); 5) when observed carrier frequencies were restricted to mainly Ashkenazi Jewish and other non-Hispanic Caucasian couples and corrected for each trial's reported proportion of mutations identified, they were 1 in 26 overall and 1 in 27 in United States trials (consistent with expectations for Caucasian populations of northern European heritage); 6) $93 \%$ of couples with positive screening results chose to have counseling and prenatal diagnosis; and 7) $88 \%$ of couples (21/24) with a fetus homozygous for cystic fibrosis chose termination (100\% in Europe and 63\% in the United States). It was recognized that the information gathered from these screening trials might not be representative of the decision-making processes in other racial/ethnic groups.

\section{LOGISTICS OF SAMPLING}

Two-step screening can be successfully done using either blood $^{5,15-20,22,23}$ or buccal $1^{5,7,10-12,17,20-22}$ samples. One-step screening, however, is most effectively accomplished using buccal cells collected by brush, "scoop," or mouthwash. ${ }^{5-14}$ This method of collection is inexpensive and is well suited to collecting samples in primary care settings or at home. Buccal samples are stable when shipped at ambient temperature, and testing has been successfully performed on buccal lysates stored frozen for 3 to 4 years (personal communications, $\mathrm{L}$. A. Bradley, K. W. Klinger, W. W. Grody). Buccal sample failure rates are generally $1 \%$ or less; results can nearly always be obtained from blood samples. When repeat samples are needed, they can nearly always be obtained. Blood spots on filter paper cards have also been used as a sampling method, ${ }^{24}$ but details of performance have not been reported.

\section{CURRENT AND FUTURE TESTING METHODOLOGIES}

Of the wide variety of testing methodologies available, nearly all screening trials have chosen forward dot-blot, reverse dotblot, or $\mathrm{ARMS}^{\mathrm{TM}}$ (amplification refractory mutation system) technologies. All appear reliable, require only a small capital investment, support a reasonable throughput of samples, can test either purified DNA or buccal lysates, and require only a moderate level of technical skill. Current estimates of unit reagent costs are about $\$ 30$ or less per test. In the United States, however, no kits have been approved by the Food and Drug Administration for cystic fibrosis testing and none is known to be under review. Laboratories offering such testing will likely come under regulations for "home brew" or, possibly, analyte specific reagents. More automated methodologies are being developed; these may improve capacity and throughput and reduce some costs (e.g., less technologist time) but may require significant capital investment. Examples include: $\mathrm{ARMS}^{\mathrm{TM}}$-based fluorescent systems (Zeneca Diagnostics, MacCheshire, UK), automation of reverse dot-blot strips (Roche Molecular Systems, Alameda, CA), micro-arrays (Affymetrix GeneChip, Santa Clara, CA), and CMST $^{\mathrm{TM}}$ (cleavable mass spectrometry technology, Rapigene/Chiroscience, Bothell, WA). During the Scarborough Conference, there was considerable discussion about the economics of screening versus diagnostic testing, and the diagnostics manufacturers are now more aware that there is a limit to the justifiable expenditure for reagents per screening test.

\section{ECONOMIC ANALYSIS}

Economic analysis is undertaken to inform policy-making, a process that must take into account a variety of medical, social, and ethical perspectives. Two economic analyses were presented and discussed. The first analysis reported costs based on a single program's experience, calculated the cost per case voluntarily averted and performed a quality of life analysis. ${ }^{25}$ The second analysis reported cost per case detected and based its calculations on summary estimates of participation rates and choices that couples made at each step of the screening process (derived from published trials). ${ }^{18,26-38}$ Both analyses considered the following as costs: 1) educating the couple and offering the test; 2 ) obtaining the samples; 3 ) performing/interpreting the laboratory test; 4) counseling those identified to be at risk; 5) performing the diagnostic testing; and 6) performing selective terminations of affected pregnancies for those who choose that option. The principal cost savings would result from reduced lifetime medical costs directly associated with treatment. This is currently estimated, with discounting, to be at least $\$ 600,000$. ${ }^{1,25}$ There was general concern that some might misinterpret performing economic analyses to mean that the sole aim of screening is saving healthcare dollars through termination of affected fetuses rather than providing couples with a choice. However, some participants felt that such analyses do have value in guiding rational implementation.

Using a range of program parameters (e.g., uptake rate and amniocentesis acceptance rate), the cost per cystic fibrosis case prenatally identified is estimated to be between $\$ 300,000$ and $\$ 500,000$ (cost per case avoided is similar due to the observed high selective termination rate). These estimates appear not to be influenced greatly by screening model (one-step versus twostep) or sample type (buccal versus blood). Screening uptake, partner participation and amniocentesis acceptance rates could influence the costs substantially if they were to be low. However, data from published trials show these rates to be uniformly high. The estimates are sensitive to the cost of laboratory testing; cost per case detected increases by about $\$ 100,000$ when the laboratory component (number 3 above) increases from $\$ 30$ to $\$ 60$. Overall, it was found that laboratory testing is likely to account for approximately $50 \%$ of the costs of cystic fibrosis screening. The remaining costs include: patient education/test offering $(20 \%)$, program management $(12 \%)$, sample collec- 
tion/transport (10\%), genetic counseling and diagnostic testing (4\%), and other costs (4\%).

Similar types of analysis indicate that screening costs for open neural tube defects/Down syndrome are as high as $\$ 100,000$ per case detected. This is roughly one-third the cost per case detected for cystic fibrosis. The long-term costs of cystic fibrosis screening may, however, be lower (by somewhat less than a factor of two), ${ }^{28}$ because subsequent pregnancies do not require additional testing if the partners remain together. It has been suggested that "cascade testing" of a carrier's relatives might also reduce the cost per case detected, ${ }^{39}$ but published data indicate that such reductions would be small. ${ }^{40}$ Published costs per case detected reported from individual studies range from $\$ 50,000$ to $\$ 1,050,000$ (after conversion to 1998 dollars). Both of the extremes can largely be attributed to the expense of the laboratory component ( $\$ 6$ and $\$ 150$, respectively). ${ }^{17,25,28,31,36}$

\section{KEY ISSUES}

The formal presentations constituted the basis for identifying key issues, which were then addressed in some detail by the participants in working groups. The following is a summary of those deliberations.

\section{Does prenatal screening for cystic fibrosis meet established screening criteria?}

The group agreed that prenatal cystic fibrosis screening satisfies all of the requirements of a worthwhile screening program. ${ }^{41}$ Cystic fibrosis is a well-defined disorder with a known prevalence. Although phenotypic variability does exist, nearly all affected individuals have substantial multi-system disease with a well-understood pattern of clinical consequences. Therapeutic regimens have steadily improved, but a cure is not anticipated in the near future. The only alternative option currently available during pregnancy is prenatal diagnosis with the possibility of termination. Based on the intervention trials and the number of laboratories currently providing DNA testing, ${ }^{42}$ the group concluded that facilities to support prenatal cystic fibrosis screening can be readily developed. Additional genetic counselors might be needed to support the two-step screening model. Similar prenatal screening and diagnostic programs have been shown to be ethically acceptable to most healthcare professionals and patients. The screening test itself is simple, noninvasive, reliable, and safe. Effective diagnostic testing is available. Costs are not excessive. Screening trials have shown test performance to be acceptable in Ashkenazi Jewish and other non-Hispanic Caucasian populations.

\section{Is it ethical to offer prenatal screening for cystic fibrosis routinely?}

The group addressed concerns that have been voiced about cystic fibrosis prenatal screening and concluded that, although important, these concerns are not different from those that apply generally to prenatal screening and DNA-based testing. Examples of such concerns include: 1) whether the option of termination devalues the lives of affected individuals or impedes the search for a cure, and 2) whether confidentiality of results can be ensured and discrimination prevented. The conference participants felt that these concerns warranted continued consideration. They should not, however, block implementation of screening programs, thereby removing the couple's option to decide about testing. The group consensus was that prenatal screening for cystic fibrosis should proceed, once an individual program has adequate facilities in place for education, testing, counseling, quality control, and monitoring.

\section{Should the initial screening test be an inquiry about racialethnic heritage?}

Both the population prevalence of cystic fibrosis and the frequency of identifiable mutations vary greatly depending upon race and ethnicity. ${ }^{1}$ Ashkenazi Jewish and other non-Hispanic Caucasian populations have the highest prevalences, followed by Hispanic, African American, and Asian American populations. Cystic fibrosis testing has the highest detection rate in Ashkenazi Jews, followed by other non-Hispanic Caucasians, African Americans, Hispanics, and Asian Americans. One way to understand the impact of population prevalence and percentage of identifiable mutations is to determine the number of couples screened per case detected. It would be necessary to screen 3500 non-Hispanic Caucasian couples to identify one case of cystic fibrosis [population prevalence divided by the product of the proportion of identifiable mutations in the two partners, or $\left.2500 /(0.85)^{2}\right]$. For Ashkenazi Jewish, Hispanic, African American, and Asian American populations, one case would be identified for every $2,800,24,000,27,000$ and 333,000 couples screened, respectively. ${ }^{32}$ If the allowable cost to identify each case were arbitrarily set at $\$ 400,000$, as much as $\$ 110$ could be spent to screen a non-Hispanic Caucasian couple. Using the same methodology, the maximum allowable unit test costs per couple for Ashkenazi Jews, Hispanics, African Americans, and Asian Americans are $\$ 140, \$ 17, \$ 15$, and $\$ 1$, respectively. ${ }^{32}$ It was agreed that prenatal screening for cystic fibrosis may need to employ a preliminary screening question to identify specific population groups eligible for screening, as is now the accepted practice for some other genetic disorders, such as Tay-Sachs disease. The implementation of such a strategy was acknowledged by some to be difficult from a sociological perspective, and this concern prevented complete agreement. In spite of this, the group agreed that any population with a detectable cystic fibrosis carrier rate of 1 in 35 or greater should be offered routine prenatal screening. According to this guideline, such testing should currently be offered to Ashkenazi Jewish and other nonHispanic Caucasian couples. In a couple with mixed ethnic/racial background, testing could be offered as long as one of the partners belongs to the group at risk.

\section{How many mutations should be included in a testing panel?}

Participants reviewed mutation panels currently in use $\mathrm{e}^{42}$ and considered how to determine whether a mutation should be included in a cystic fibrosis screening panel. Suggested criteria 
were that the mutation must have a measurable frequency (at least $0.1 \%$ ) and must have been shown to be associated with significant morbidity and a high risk of premature death. ${ }^{43}$ It is likely that 20 to 24 mutations would satisfy these criteria for Ashkenazi Jewish and other non-Hispanic Caucasian populations. As an alternative approach, it was agreed that a program could utilize fewer mutations (which satisfy the above criteria) if the program could document a carrier detection rate of at least 1 in 35 in the population served. There was discussion about the appropriateness of including mutations associated with more variable or "less severe" phenotypes (e.g., mutations associated with congenital bilateral absence of the vas deferens); identification of such mutations in pregnancy can create complex counseling situations for genetic service providers. Recommendations on this issue were beyond the scope of this meeting and will be addressed by the joint American College of Medical Genetics/American College of Obstetricians and Gynecologists task force on cystic fibrosis testing. Diagnostics manufacturers voiced the need for expert opinion in developing products supporting prenatal screening for cystic fibrosis.

\section{Should one screening model be recommended?}

Two models have been successfully used (Figure 1). The onestep model has a lower "false positive" rate, ${ }^{*}$ while maintaining the same detection as the two-step model. This reduces the number of women who need to be recalled for genetic counseling and consideration of further testing. Genetic counseling is limited to the 289 screen positive couples, and a diagnostic option is available to all of them. Seventy-two affected fetuses are identified. Some participants felt that not informing women (or their partners) of their carrier status was an important disadvantage, with potential ethical and legal ramifications. There was, however, agreement that additional advantages of one-step screening include reducing stigmatization and associated difficulties with insurability and fostering a feeling of unity between partners.

The two-step model provides information about individual carrier status. When only a woman is found to have an identifiable mutation, the couple is given a residual risk for having an affected child, but it is not possible to offer definitive diagnostic testing. Two intervention trials that used the two-step model demonstrated initially increased levels of maternal anxiety that declined rapidly when the partner's negative result was received, ${ }^{21,44}$ whereas two other trials did not find this initial increase. ${ }^{16,18}$ Two-step screening avoids the need to obtain samples from about $97 \%$ of the partners, thereby simplifying the logistics of recruitment. In this model, 8500 women (Figure 1) receive counseling and the same 72 affected fetuses are identified. It was speculated that the woman's knowledge about carrier status, if shared with family members, could lead to increased acceptance of testing in these relatives. Participants did not reach consensus upon a preferred model.

*As stated earlier, the goal of prenatal cystic fibrosis screening is the detection of an affected fetus. In screening parlance, a "false positive" result occurs when it is necessary to inform the screened individual about a positive test result, but the disorder being screented for is subsequently found not to be present.

\section{Would a reasonable alternative model be one in which both the woman's and her partner's samples are collected, tested, and carrier results routinely reported for both?}

Briefly discussed was the possibility of an alternative model in which both the woman's and partner's samples were routinely tested. This is distinctly different from the one-step (or "couple") model. It was pointed out that the time and expense of collecting the partners' samples had already been incurred as part of a one-step model. Were both partners' samples to be tested routinely, the cost of laboratory testing would double, but there would be no increase in detection of affected fetuses (even though nearly double the number of carrier individuals would be identified). The associated genetic counseling load would also increase to about $7 \%$ of couples tested (from $0.1 \%$ with the couple model, or from $3.4 \%$ with the two-step model). There was general agreement that the increased cost associated with this modification outweighed the benefit.

\section{Who will address minimum laboratory standards for a screening program?}

A joint task force of the American College of Medical Genetics and the American College of Obstetricians and Gynecologists has been charged with making specific recommendations about minimum standards for personnel and testing in laboratories performing prenatal screening for cystic fibrosis. Since it is likely that some laboratories inexperienced in DNA testing will want to implement screening, the task force will address issues that include: 1) minimum standards for laboratory quality control and quality assurance; 2 ) composition of mutation testing panels; 3) qualifications for laboratory directors and personnel; 4) content of test requisitions and reports; and 5) enhancing currently available external proficiency testing.

\section{How will healthcare providers be educated about prenatal screening for cystic fibrosis?}

It is reasonable to assume that information about prenatal screening for cystic fibrosis will be disseminated via materials and practice guidelines from professional organizations, onsite training, clinical support by local genetic service providers, and scientific meetings. The most effective of these methods for producing change in provider practice will utilize the concept of "the teachable moment" (i.e., appropriate, pertinent information that is readily available for the provider at the time an issue arises with a patient) ${ }^{45}$ More traditional approaches to continuing medical education, such as hospital rounds, seminars, and lectures have been shown to be less effective. ${ }^{46}$ One efficient way of expediting the flow of information would be to use a familiar delivery system, such as an existing prenatal screening program.

\section{How can appropriate information about cystic fibrosis and available testing be presented to patients?}

Based on experience from intervention trials, most patients appear to understand appropriate information presented in written materials (brochures/pamphlets), oral presentation, 
and/or videotape, followed by verbal interaction with clinical staff (including nurses, mid-level practitioners, and case managers).$^{5,16,18}$ Brochures have the advantages of being familiar to patients, inexpensive and relatively simple to produce, and easily translated into multiple languages. Of greater importance, appropriate field-tested brochures are already available. Two disadvantages are that brochures are less useful in individuals or populations with low literacy, and that completeness and accuracy of many existing brochures have not been directly evaluated. Although improvements can certainly be made, the materials currently available are sufficient in content, but care must be taken to ensure a balanced presentation. ${ }^{47}$

Oral presentations have the advantages of soliciting patient questions and facilitating assessment of understanding. They are also associated with high patient satisfaction. The disadvantages include a higher cost to convey an equivalent amount of information, variability in informational content, and a higher likelihood of being directive. Educational videotapes, particularly those using graphics or animation, provide a uniform (potentially multilingual) presentation of information that is attractive, holds the attention of the viewer, and reaches low literacy individuals. This approach can be efficient (e.g., minimizes provider time), but the initial production costs are higher, and not all providers have the necessary equipment and dedicated space for viewing. Audiotapes, computer interactive modules, or Internet access to information might also prove effective but have not been formally assessed and are not generally available.

\section{How will consent be obtained?}

When prenatal screening for cystic fibrosis is in the research stage, "written informed consent" is, as a rule, required. There was consensus, however, that when screening becomes routine, the same standard should be applied to obtaining consent as is in place for other screening tests (documented oral consent). This is best carried out by healthcare providers. Emphasis needs to be placed on ensuring adequate patient education so that couples can make informed decisions. Conference participants agreed that a balanced presentation was critical in the consent process.

\section{CONCLUSIONS}

Sufficient information is available from intervention trials to proceed, on a rational basis, with implementation of routine prenatal cystic fibrosis screening. All of the basic criteria for a successful screening program are satisfied. Screening costs are not excessive using either the one-step or two-step screening model, buccal or blood samples, and a variety of testing methodologies. Validated materials exist to support patient and provider education. Ethical issues are similar to those found in other forms of prenatal screening and genetic testing already routinely available. Once any given program has established adequate facilities for education, testing, counseling, and quality control and monitoring, it is acceptable for it to begin offering prenatal screening for cystic fibrosis.

\section{Acknowledgments}

We thank Joanne Beaudoin for her help in organizing and managing the meeting, and Dr. Helen Lyon for assisting with recording comments from meeting participants. Additional participants include: Garry R. Cutting, MD (Johns Hopkins School of Medicine, Baltimore, Maryland). Funding was provided by Zeneca Diagnostics, Cheshire, England; The March of Dimes Birth Defects Foundation, White Plains, New York; Genzyme, Framingham, Massachusetts; Maine Department of Human Services, Bureau of Health, Augusta, Maine; the New England Regional Genetics Group (MCJ-241003), Bar Harbor, Maine; Roche Diagnostics, Alameda, California; and Rapigene (Chiroscience), Bothell, Washington.

\section{References}

1. NIH Consensus Development Conference Statement. Genetic testing for cystic fibrosis. Available at http://odp.od.nih.gov/consensus/statements/cdc/106/106_stmt.html. Accessed June 3, 1997.

2. Holmes LB, Pyeritz RE (for the Clinical Practice Committee of the American College of Medical Genetics). Screening for cystic fibrosis. JAMA 1998;279:1068-1069.

3. American College of Obstetricians and Gynecologists Statement on Cystic Fibrosis Testing. American College of Obstetricians and Gynecologists, 1998, Washington, D.C.

4. Wald NJ. Couple screening for cystic fibrosis. Lancet 1991;338:1318-1319.

5. Brock DJH. Prenatal screening for cystic fibrosis: 5 years' experience reviewed. Lancet 1996;347:148-150.

6. Livingstone J, Axton RA, Gilfillan A, Mennie M, Compton M, Liston WA, Calder $A A$, Gordon AJ, Brock DJ. Antenatal screening for cystic fibrosis: A trial of the couple model. BMJ 1994;308:1459-1462.

7. Miedzybrodzka ZH, Hall MH, Mollison J, Templeton A, Russell IT, Dean JCS, Kelly KF, Marteau TM, Haites NE. Antenatal screening for carriers of cystic fibrosis: Randomised trial of stepwise $v$ couple screening. BMJ 1995;310:353-357.

8. Wald NJ, George L, Wald N, MacKenzie IZ. Further observations in connection with couple screening for cystic fibrosis. Prenat Diagn 1995;15:589-590.

9. Wald NJ, George LM, Wald NM. Couple screening for cystic fibrosis. Lancet 1993:342:1307-1308.

10. Harris H, Scotcher D, Hartley N, Wallace A, Craufurd D, Harris R. Cystic fibrosis carrier testing in early pregnancy by general practitioners. $B M J$ 1993;306:1580-1583.

11. Harris H, Scotcher D, Hartley N, Wallace A, Craufurd D, Harris R. Pilot study of the acceptability of cystic fibrosis carrier testing during routine antenatal consultations in general practice. Br J Gen Pract 1996;46:224-227.

12. Hartley NE, Scotcher D, Harris H, Williamson P, Watlace A, Craufurd D, Harris R. The uptake and acceptability to patients of cystic fibrosis carrier testing offered in pregnancy by the GP. J Med Genet 1997;34:459-464.

13. Doherty RA, Palomaki GE, Kloza EM, Erickson JL, Haddow IE. Couple-based prenatal screening for cystic fibrosis in primary care settings. Prenat Diagn 1996; 16:397-404.

14. Bradley LA, Johnson DD, Doherty RA, Palomaki GE, Haddow JE. Routine prenatal cystic fibrosis screening in primary care offices. Am J Hum Genet 1998;63:A13.

15. Schwartz M, Brandt NJ, Skovby F. Screening for carriers of cystic fibrosis among pregnant women: A pilot study. Eur J Hum Genet 1993;1:239-244.

16. Witt DR, Schaefer C, Hallam P, Wi S, Blumberg B, Fishbach A, Holtzman J, Kornfeld S, Lee R, Nemzer L, Palmer R. Cystic fibrosis heterozygote screening in 5,161 pregnant women. Am J Hum Genet 1996;58:823-835.

17. Mennie ME, Gilfillan A, Compton M, Curtis L, Liston WA, Pullen I, Whyte DA, Brock DJ. Prenatal screening for cystic fibrosis. Lancet 1992;340:214-216.

18. Loader S, Caldwell P, Kozyra A, Levenkron JC, Boehm CD, Kazazian HH Jr, Rowley PT. Cystic fibrosis carrier population screening in the primary care setting. Am J Hum Genet 1996;59:234-247.

19. Eng CM, Schechter C, Robinowitz J, Fulop G, Burgert T, Levy B, Zinberg R, Desnick RJ. Prenatal genetic carrier testing using triple disease screening. JAMA 1997;278:1268-1272.

20. Cuckle H, Quirke P, Sehmi I, Lewis F, Murray J, Cross D, Cuckle P, Ozols B. Antenatal screening for cystic fibrosis. Br J Obstet Gynaecol 1996;103:795-799.

21. Grody WW, Dunkel-Schetter C, Tatsugawa ZH, Fox MA, Fang CY, Cantor RM, Novak JM, Bass HN, Crandall BF. PCR-based screening for cystic fibrosis carrier mutations in an ethnically diverse pregnant population. Am J Hum Genet 1997;60:935-947. 
22. Brambati B, Anelli MC, Tului L. Prenatal cystic fibrosis screening in a low-risk population undergoing chorionic villus sampling for fetal karyotyping. Clin Genet 1996;50:23-27.

23. Jung U, Urner U, Grade K, Coutelie C. Acceptability of carrier screening for cystic fibrosis during pregnancy in a German population. Hum Genet 1994;94:19-24.

24. Clayton EW, Hannig VL, Pfotenhauer JP, Parker RA, Campbell PW, Phillips IA. Teaching about cystic fibrosis carrier screening by using written and video information. Am J Hum Genet 1995;57:171-281.

25. Rowley PT, Loader S, Kaplan RM. Prenatal screening for cystic fibrosis carriers: An economic evaluation. Am J Hum Genet 1998;63:1160-1174.

26. Asch DA, Patton IP, Hershey JC, Mennuti MT. Reporting the results of cystic fibrosis carrier screening. Am J Obstet Gynecol 1993;168:1-6.

27. Brock DJH. Realistic cost must be established for genetic counseling in two-step screening. BMJ 1996;312:908.

28. Cuckle HS, Richardson GA, Sheldon TA, Quirke P. Cost effectiveness of antenatal screening for cystic fibrosis. BMJ 1995;311:1460-1463.

29. Cystic fibrosis and DNA tests: implications of carrier screening. Office of Technology Assessment. Pages 213-226. 1992 (OTA-BA-532), NTIS No. PB92-216142, GPO No. 052-003-01291-0.

30. Garber AM, Fenerty JP. Costs and benefits of prenatal screening for cystic fibrosis. Med Care 1991;29:473-489.

31. Ginsberg G, Blau H, Kerem E, Springer C, Kerem B, Akstein E, Greenberg A, Kolumbos A, Abeliovich D, Gazit E, Yahav Y. Cost-benefit analysis of a national screening programme for cystic fibrosis in an Israeli population. Health Econ 1994;3:5-23.

32. Haddow JE, Palomaki GE, Bradley LA, Doherty RA. Screening for cystic fibrosis. JAMA 1998;279:1068.

33. Lieu TA, Watson SE, Washington AE. The cost-effectiveness of prenatal carrier screening for cystic fibrosis. Obstet Gynecol 1994;84:903-912.

34. Miedzybrodzka Z, Semper I, Shackley P, Abdalla M, Donaldson C. Stepwise or couple antenatal carrier screening for cystic fibrosis? Women's preferences and willingness to pay. / Med Genet 1995;32:292-293.

35. Miedzybrodzka Z, Shackley P, Donaldson C, Abdalla M. Counting the benefits of screening: A pilot study of willingness to pay for cystic fibrosis carrier screening. J Med Screen 1994;1:82-83.

36. Morris JK, Oppenheimer PM. Cost comparison of different methods of screening for cystic fibrosis. J Med Screen 1995;2:22-27.

37. Robson M, Abbott J, Webb K, Dodd M, Walsworth-Bell J. A cost description of an adult cystic fibrosis unit and cost analysis of different categories of patients. Thorax 1992;47:684-689.

38. Wilfond BS, Fost $\mathrm{N}$. The cystic fibrosis gene: Medical and social implications for heterozygote detection. JAMA 1990;263:2777-2783.

39. Super M, Schwartz MJ, Malone G, Roberts T, Haworth A, Dermody G. Active cascade testing for carriers of cystic fibrosis genes. BMJ 1994;308:1462-1468.

40. Holloway S, Brock DJ. Cascade testing for the identification of carriers of cystic fibrosis. J Med Screen 1994;1:159-164.

41. Wald N, Cuckle H. Reporting the assessment of screening and diagnostic tests Br J Obstet Gynecol 1989;96:389-396.

42. Grody WW, Desnick RJ, Carpenter NJ, Noll WW. Diversity of cystic fibrosis mutation-screening practices. Am J Hum Genet 1998;62:1252-1254.

43. Rosenstein BJ, Cutting GR. The diagnosis of cystic fibrosis: A consensus statement. J Pediatr 1998;132:589-595.

44. Mennie ME, Compton ME, Gilfillan A, Brock DJH. Prenatal screening for cystic fibrosis: Psychological effects on carriers and their partners. J Med Genet 1993;30:543-548.

45. Leist JC, Kristofco RE. The changing paradigm for continuing medical education: Impact of information on the teachable moment. Bull Med Libr Assoc 1990;78:173-179.

46. Davis $\mathrm{D}$, Thomson $\mathrm{MA}$, Oxman $\mathrm{AD}$, Haynes $\mathrm{B}$. Changing physician performance. JAMA 1995;274:700-705.

47. Loeben GL, Marteau TM, Wilfond BS. Mixed messages: Presentation of information in cystic fibrosis-screening pamphlets. Am J Hum Genet 1998;63:1181-1189. 